La recherche en environnement : Apprendre à oeuvrer dans la diversité et le croisement des savoirs | Compte-rendu

(2) OpenEdition

Journals

Édition électronique

URL : http://journals.openedition.org/ere/860

DOI : 10.4000/ere.860

ISSN : 2561-2271

Éditeur

Centr'ERE

Référence électronique

«La recherche en environnement: Apprendre à oeuvrer dans la diversité et le croisement des savoirs |

Compte-rendu », Éducation relative à l'environnement [En ligne], Volume 11 | 2014, mis en ligne le 20

décembre 2013, consulté le 24 septembre 2020. URL : http://journals.openedition.org/ere/860 ; DOI :

https://doi.org/10.4000/ere.860 


\section{La recherche en environnement : Apprendre à oeuvrer dans la diversité et le croisement des savoirs | Compte-rendu}

Colloque étudiant - Université du Québec à Montréal - 15 mai 2013

2 Organisé par les associations étudiantes de la maîtrise et du doctorat en sciences de l'environnement de l'Université du Québec à Montréal (UQAM) le 15 mai 2013, ce colloque Apprendre à oeuvrer dans la diversité et le croisement des savoirs s'est inscrit dans le contexte des 40 e et $25 \mathrm{e}$ anniversaires des programmes de maîtrise et de doctorat en sciences de l'environnement, marqués par des débats autour de l'institutionnalisation de l'interdisciplinarité de la recherche et de la formation en environnement.

3 Un groupe d'étudiants et étudiantes de l'Institut des sciences de l'environnement (ISE) et membres du Centr'ERE est à l'initiative d'une telle rencontre, qui visait à créer un espace convivial de réflexion et d'échanges à propos des défis que pose le croisement des savoirs en sciences de l'environnement.

4 Le colloque s'est articulé autour de trois questions phares, invitant les intervenants à : identifier les croisements de savoirs (multi, inter, trans ou in-disciplinaires) à l'oeuvre dans les recherches présentées ; mettre en relief les curiosités, possibilités et défis engendrés par ces métissages et entrevoir des stratégies pour faire face aux enjeux rencontrés dans le contexte des projets de recherche en sciences de l'environnement.

Lucie Sauvé a prononcé la conférence d'ouverture du colloque, proposant un ensemble de repères épistémologiques et de considérations éthiques et stratégiques au sujet du dialogue des savoirs en environnement. Trois principaux thèmes ont été abordés dans cette présentation: le réseau notionnel de l'interdisciplinarité, les dimensions cognitive et pragmatique du travail interdisciplinaire, les voies de construction et de légitimation du savoir environnemental, essentiellement transdisciplinaire, à travers l'interfécondation des savoirs de divers types. La chercheure a invité les participants à adopter une posture de réflexivité permanente à l'égard de la science et de la recherche 
puisqu'elles sont tributaires de discours et de pratiques socialement constitués et situés.

6 Le colloque s'est ensuite poursuivi avec les communications, individuelles ou par équipes, de près de 30 étudiants et étudiantes. Le comité organisateur proposait cinq "points de convergence» en fonction des propositions reçues: santé environnementale, milieux riverains et côtiers, questions culturelles, milieux urbains et enjeux politiques. Différents enjeux de la pratique et de l'application de l'interdisciplinarité ont été soulevés. Les participants ont souligné entre autres le temps et l'énergie requis par la pratique interdisciplinaire du croisement des savoirs, particulièrement lors des processus collaboratifs entre chercheurs: prendre des décisions concertées, élaborer des questionnements communs, réfléchir à des types de données complémentaires, s'assurer de bien définir les frontières et les espaces de partage, choisir les variables pertinentes, naviguer dans le croisement de méthodologies qualitatives et quantitatives...

7 Une intervenante a soutenu par ailleurs que le croisement des savoirs rend le chercheur à la fois fragile et plus fort. En effet, il doit sortir de sa «zone de confort disciplinaire ", se mettre en situation de vulnérabilité et peut aller jusqu'à se sentir quelque peu «imposteur» quand vient le temps de porter des savoirs interdisciplinaires construits collectivement dans des revues scientifiques ou demandes de financements, qui ne supportent encore que trop rarement les projets de recherche interdisciplinaires. D'un autre côté, la diversité de regards renforce un certain pouvoir d'action et de création en termes de solutions et contribue à donner une signification plus grande au projet de recherche. Ces éléments témoignent de cette réflexivité requise pour un chercheur qui s'engage dans l'interdisciplinarité, mais aussi des tensions interdisciplinaires qui vont jusqu'à mettre en question la légitimité de ses savoirs.

8 Au fil des différents projets de recherche présentés, les participants ont évoqué des valeurs et des principes caractéristiques de la pratique interdisciplinaire, telle une attitude d'ouverture, d'humilité, d'écoute et de créativité. Ils ont également reconnu que le croisement des pratiques et des savoirs invite à sortir des processus habituels, à transgresser les frontières établies par les schèmes et référents qui organisent les disciplines canoniques.

9 En clôture, un panel de discussion regroupant chercheurEs, professeurEs, étudiantEs et praticienNEs des sciences de l'environnement a permis de dégager des éléments de synthèse de la journée et de poursuivre plus loin la réflexion. La présence d'étudiantEs et d'enseignantEs cheminant depuis plusieurs années au sein de programmes de formation en sciences de l'environnement a ajouté une perspective historique, dont a résulté une question centrale, formulée en fin de colloque, à partir d'une proposition de la conférence initiale : à l'image de ce l'on nomme environmental science dans le monde anglophone, peut-on considérer aujourd'hui en francophonie que, des sciences de l'environnement, serait née la science de l'environnement comme discipline intégrée? Quelle serait alors la place des savoirs dits indisciplinaires, fugitifs, en dehors des zones de contrôles?

Dans un séminaire international sur l'interdisciplinarité dans les universités organisé en septembre $1970^{1}$ qui nourrît les réflexions et définitions à l'origine de la création de l'ISE, la transdisciplinarité se définissait comme "une axiomatique commune à un ensemble de disciplines", autrement dit une science fondée sur une série d'axiomes 
communs, de principes et de valeurs avec un caractère d'évidence pour les disciplines qui les partagent. Un horizon pour les sciences de l'environnement? De tels questionnements pourraient inspirer un prochain groupe d'étudiants-chercheurs à poursuivre la réflexion collective dans le cadre d'un événement semblable, c'est-à-dire la création d'un espace de dialogue récurrent, qui favorise cette réflexivité permanente essentielle à la science et à la recherche interdisciplinaire.

Le comité organisateur du colloque :

Félix Lebrun-Paré, Hugue Asselin, Laurence Brière, Anne-Isabelle Cuvillier, Clara Munoz et Anne Samson. Rédigé avec la collaboration de Jérôme Lafitte.

\section{NOTES DE FIN}

1. Apostel L., Berger A., Briggs A. et Michaud G. (dir.) (1972). L'interdisciplinarité. Problèmes d'enseignement et de recherche dans les universités. Rapport du séminaire sur l'interdisciplinarité dans les universités organisé par le CERI, le ministère français de l'Éducation et l'Université de Nice du 7 au 12 septembre 1970. Paris : OCDE. 